\title{
Concurrence Percolation in Quantum Networks
}

\author{
Xiangyi Meng $\odot{ }^{1,4}$ Jianxi Gao $\odot,{ }^{2, *}$ and Shlomo Havlin ${ }^{3,1, \dagger}$ \\ ${ }^{1}$ Center for Polymer Studies and Department of Physics, Boston University, Boston, Massachusetts 02215, USA \\ ${ }^{2}$ Department of Computer Science, Rensselaer Polytechnic Institute, Troy, New York 12180, USA \\ ${ }^{3}$ Department of Physics, Bar-Ilan University, 52900 Ramat-Gan, Israel \\ ${ }^{4}$ Center for Complex Network Research and Department of Physics, Northeastern University, Boston, Massachusetts 02115, USA
}

(Received 13 July 2020; accepted 24 March 2021; published 27 April 2021)

\begin{abstract}
Establishing long-distance quantum entanglement, i.e., entanglement transmission, in quantum networks (QN) is a key and timely challenge for developing efficient quantum communication. Traditional comprehension based on classical percolation assumes a necessary condition for successful entanglement transmission between any two infinitely distant nodes: they must be connected by at least a path of perfectly entangled states (singlets). Here, we relax this condition by explicitly showing that one can focus not on optimally converting singlets but on establishing concurrence-a key measure of bipartite entanglement. We thereby introduce a new statistical theory, concurrence percolation theory (ConPT), remotely analogous to classical percolation but fundamentally different, built by generalizing bond percolation in terms of "sponge-crossing" paths instead of clusters. Inspired by resistance network analysis, we determine the path connectivity by series and parallel rules and approximate higher-order rules via star-mesh transforms. Interestingly, we find that the entanglement transmission threshold predicted by ConPT is lower than the known classical-percolation-based results and is readily achievable on any series-parallel networks such as the Bethe lattice. ConPT promotes our understanding of how well quantum communication can be further systematically improved versus classical statistical predictions under the limitation of QN locality-a "quantum advantage" that is more general and efficient than expected. ConPT also shows a percolationlike universal critical behavior derived by finite-size analysis on the Bethe lattice and regular two-dimensional lattices, offering new perspectives for a theory of criticality in entanglement statistics.
\end{abstract}

DOI: 10.1103/PhysRevLett.126.170501

Recently, much attention has been given to quantum network (QN) [1] (sometimes also referred to as the quantum Internet [2]) for better understanding of entanglement transmission, i.e., establishing long-distance entanglement between arbitrary two nodes, as a quantum information flow from the perspective of network science [3]. Only local operations and classical communication, a.k.a. LOCC [4] are allowed between different nodes in a QN-a limitation by locality. In this Letter, we focus on a minimal version of $\mathrm{QN} \mathrm{[1]} \mathrm{that} \mathrm{is} \mathrm{an} n$-node network, denoted $\mathcal{G}_{\theta}(n)$. Each link $i$ is an identical pure state $\left|\psi_{i}(\theta)\right\rangle=\cos \theta|00\rangle+\sin \theta|11\rangle$ weighted by the sole parameter $0 \leq \theta \leq \pi / 4$ that admits a probability measure $p:=2 \sin ^{2} \theta$ known as the optimal probability to convert $\left|\psi_{i}(\theta)\right\rangle$ to a singlet (i.e., a maximally entangled state by $\theta=\pi / 4)$. Hence, a mapping between entanglement transmission in infinite QN and classical bond percolation theory, called classical entanglement percolation (CEP) has been discovered [1]. This indicates the existence of a nontrivial threshold —in terms of $p$ per link-for establishing sufficient entanglement between arbitrary two nodes. Interestingly, a scheme called quantum entanglement percolation (QEP) [1] shows that there are scalable quantum strategies that can change the whole network topology and thus may lower the classical percolation threshold, suggesting a "quantum advantage" vs CEP for specific network topologies. Generalizations to mixed states [5], tripartite entanglements (GHZ states) [6], and random networks [7-9] have since been studied under the QEP scheme for further efforts on lowering the threshold, in hope of exploiting more advantage until reaching some presumed minimum threshold [6].

Still, all aforementioned schemes are based on the classical percolation framework. Thus, no matter how designed, the schemes have always demanded one condition to achieve entanglement transmission in infinite QN: two infinitely distant nodes must be connected by at least one path of singlets, so that by applying a specific LOCC called "swapping" [10] at in-between nodes, a singlet can eventually be established between the pair of nodes [1]. Naturally, a fundamental question whether, in general, such a condition can be relaxed arises $[6,11]$. The inability of answering this within the classical percolation framework (since the question is pertinent to the mapping itself) substantially prevented us from a true comprehension of the quantum advantage possessed by different QN topology. Simply adding a nonscalable quantum strategywhich can only change the network topology locally-into 
TABLE I. ConPT predicts the lowest threshold compared to those obtained from known classical-percolation-theory-based schemes. All thresholds are given in $\theta$ under a change of variables $p \equiv 2 \sin ^{2} \theta$.

\begin{tabular}{lclcc}
\hline \hline [unit: $\left.(\pi / 4)^{-1} \theta\right]$ & Bethe lattice (degree $k)$ & Square & Honeycomb & Triangular \\
\hline CEP [1] & $(4 / \pi) \sin ^{-1}[1 / \sqrt{2(k-1)}]$ & 0.670 & 0.777 & 0.545 \\
QEP [1,7,11] & $(4 / \pi) \sin ^{-1}{\sqrt{P_{\text {swap }}(k) / 2}}^{\text {a }}$ & 0.670 & 0.761 & 0.545 \\
QEP-GHZ [6] & $(4 / \pi) \sin ^{-1}{\sqrt{P_{\mathrm{GHZ}}(k) / 2}}^{\text {b }}$ & 0.584 & 0.745 & 0.481 \\
ConPT (Fig. 1) & $(2 / \pi) \sin ^{-1}(1 / \sqrt{k-1})$ & $0.42(8)$ & $0.51(8)$ & $0.32(8)$ \\
\hline \hline
\end{tabular}

${ }^{a} P_{\text {swap }}(k)=2 x-x^{2}$, where $x(k)$ is the solution of $2 x+x^{k}(x k-x-k-1)-(1-x) /(k-1)=0$ by the $q$-swapping strategy [7].

${ }^{\mathrm{b}} P_{\mathrm{GHZ}}(k)=x$ is the solution of $1-(1-x) \sum_{i=0}^{\lfloor k / 2-1\rfloor}\left(\begin{array}{c}2 i \\ i\end{array}\right) 4^{-i}\left(2 x-x^{2}\right)^{i}-1 /(k-1)=0$ where $\lfloor\cdot\rfloor$ is the floor function [6].

the QEP scheme is not helpful for making a statistical argument on the percolation threshold, and hence the generality of the quantum advantage on arbitrary network topology is yet to be understood.

In response to the question, here we introduce an alternative mapping called concurrence percolation theory (ConPT) which explicitly relaxes the necessity of establishing a path of singlets. We directly generalize percolation theory in terms of path connectivity and apply it to concurrence [12] (a key measure of bipartite entanglement defined as $c:=\sin 2 \theta$ for a pure state), rather than singlet conversion probability like in the traditional CEP/QEP scheme [1]. The existence of ConPT itself, as we will see, implies that entanglement transmission can also be established when the two infinitely distant nodes are connected by paths of only imperfectly entangled states - as long as there are enough paths. Interestingly, we find that the threshold predicted by ConPT is the lowest threshold compared to earlier known schemes (Table I). Our results help extending our knowledge of quantum advantage as well as discovering potentially new criticality in entanglement statistics.

Percolation as a theory of connectivity.-Recent results [8] hint that the cluster size may be an ill-defined order parameter of a genuine statistical theory of entanglement transmission. Indeed, percolation theory was initially about path connectivity before being reformulated into clusters due to mathematical convenience. Thus, instead of clusters, here we make direct use of the classical "sponge-crossing" probability $P_{\mathrm{SC}}$-the probability that there is an open path connecting two far-apart boundaries, which was used in the early studies of bond percolation on 2D (and higherdimensional) lattices [13]. $P_{\mathrm{SC}}$ can be calculated by connectivity rules using the link weights $p(0 \leq p \leq 1)$ - which are simply determined numbers before a posteriori explained as occupation probabilities-along all paths that connect the two boundaries. In the thermodynamic limit, $n \rightarrow \infty$, we expect that $P_{\mathrm{SC}}$ should approach either 0 or 1 , respectively, in the sub- or supercritical regimes, separated by the percolation threshold $p_{\text {th }}$ [13].

For a series-parallel network [14], by definition, only two connectivity rules, namely, series and parallel rules, are sufficient for calculating $P_{\mathrm{SC}}$. Surprisingly, the series and parallel rules for classical percolation are simple but both are extensible and commutable (Table II), similar to calculating the net electrical resistance in a resistance network. When "loops" exist (for example, in a bridge circuit [14]), also required are higher-order connectivity rules which are complicated (but closed form owing to the additivity of probability measure). Additionally, these rules can be well approximated by only series and parallel rules via a useful technique known as the star-mesh (SM) transform [15] (Table II), which is similar to a local renormalization group process (see Supplemental Material [16]). This technique was used in, e.g., the Frank-Lobb algorithm [17], for solving classical percolation problems.

We expect that ConPT can be built similarly, yet not on probability but on concurrence. We denote by $C_{\mathrm{SC}}$ the sponge-crossing concurrence and $c_{\text {th }}$ the corresponding threshold on the concurrence $c$ of each link in $\mathcal{G}_{\theta}(n) . C_{\mathrm{SC}}$ in the sub- or supercritical regimes should also approach either 0 or 1 in the thermodynamic limit. We proceed by examining possible connectivity rules in $\mathrm{QN}$ for transmission of concurrence that are allowed by LOCC in an optimal manner. In general, a full probabilistic argument should be built since LOCC involves selective measurements [18] of quantum states and results in probabilistic outcomes [11]. However, there is a subset of LOCC which is considered "deterministic" as it only yields one possible outcome in terms of pure states, up to unitary equivalence. The deterministic LOCC is what we need for building connectivity rules so as to keep ConPT a determined theory of connectivity and avoid mixing concurrence with probability measures. Fortunately, we find that ConPT also admits similarly simple but general series and parallel rules (Table II), the realizability of which by LOCC is discussed below.

Series and parallel rules as LOCC.-(i) Series rule. When two links of concurrence $c_{\mathrm{AR}}, c_{\mathrm{RB}}$ are connected in series between three nodes, Alice-Relay-Bob (A-R-B), "swapping" on R projects out four probabilistic outcomes between A and B [10]. The final average concurrence is $C=\sum_{k=1}^{4} \omega_{k} C_{k}$, where $\omega_{k}$ is the probability of producing a pure state of concurrence $C_{k}$. $\sum_{k} \omega_{k}=1$ is understood. When a particular Bell basis (the $X Z$ basis [11]) is chosen for projection, not only is $C$ optimal but also all $C_{k}$ are identical to the product of concurrences of the two 
TABLE II. Connectivity rules.

\begin{tabular}{lll}
\hline \hline & \multicolumn{1}{c}{ Classical } & \multicolumn{1}{c}{ ConPT } \\
\hline Series rule & $p=p_{1} p_{2} \cdots$ & $c=c_{1} c_{2} \cdots$ \\
Parallel rule & $1-p=$ & $\frac{1+\sqrt{1-c^{2}}}{2}=\max \left\{\frac{1}{2}\right.$, \\
& $\left(1-p_{1}\right)\left(1-p_{2}\right) \cdots$ & $\left.\frac{1+\sqrt{1-c_{1}^{2}}}{2} \frac{1+\sqrt{1-c_{2}^{2}}}{2} \cdots\right\}$
\end{tabular}

Higher-order Can be approximated by the SM transform by rules the following two-step argument:

1. The SM transform can reduce an $n$-graph to an $(n-1)$-graph (right panel) and is solvable by applying the series and parallel rules recursively through a group of $n(n-1) / 2$ coupled equations (see Supplemental Material [16] for details).

2. Applying the transform consecutively on a network can reduce nodes one by one-and thus reduce any topology to two nodes, yielding the final (approximate) connectivity between them (bottom panel, i. $\rightarrow$ viii.)
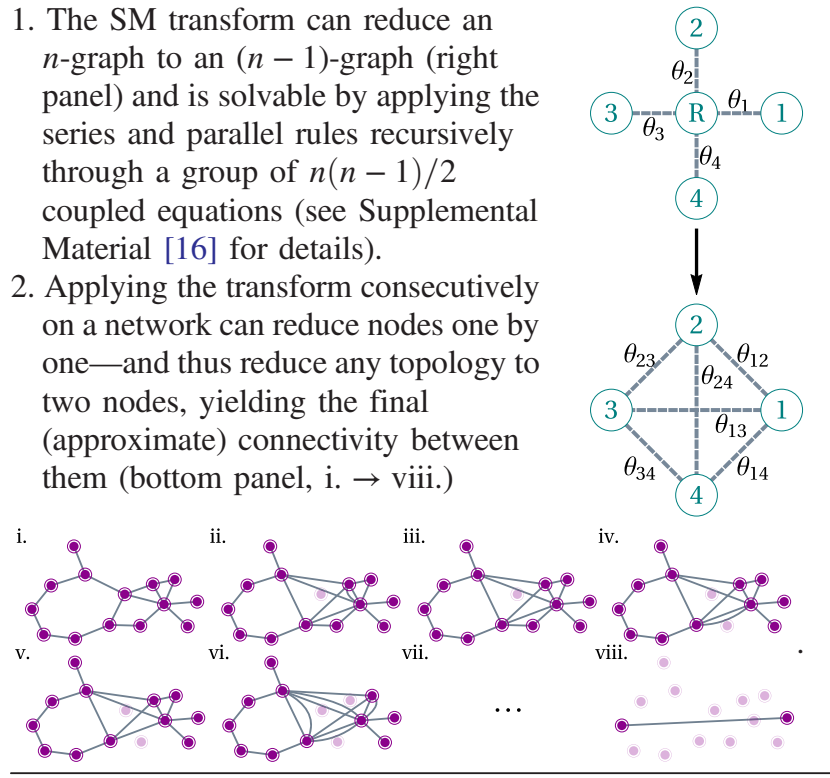

links, $C_{k}=c_{\mathrm{AR}} c_{\mathrm{RB}}$, hence admitting deterministic LOCC. (ii) Parallel rule. For two parallel links between $\mathrm{A}$ and $\mathrm{B}$, the product state $\left|\psi_{\mathrm{AB}}\left(\theta_{1}\right)\right\rangle \otimes\left|\psi_{\mathrm{AB}}\left(\theta_{2}\right)\right\rangle=\left(\cos \theta_{1}|00\rangle+\right.$ $\left.\sin \theta_{1}|11\rangle\right)\left(\cos \theta_{2}|00\rangle+\sin \theta_{2}|11\rangle\right)$ belonging to $\mathcal{H}_{1} \otimes$ $\mathcal{H}_{2}$ is a "two-ququart" state. By Nielsen's theorem [4], the maximally entangled two-qubit pure state obtainable by LOCC is $\cos \theta_{\text {tot }}|00\rangle+\sin \theta_{\text {tot }}|11\rangle$, where $\cos \theta_{\text {tot }}=$ $\cos \theta_{1} \cos \theta_{2}$ is equal to the largest Schmidt coefficient, provided that $\cos \theta_{1} \cos \theta_{2}>1 / \sqrt{2}$. When $\cos \theta_{1} \cos \theta_{2} \leq$ $1 / \sqrt{2}$, a singlet $\cos \theta_{\text {tot }}=1 / \sqrt{2}$ can always be obtained. Again, not only is the LOCC deterministic but it actually optimizes the obtainable average concurrence $C=$ $\sum_{k} \omega_{k} C_{k}$ as well, a result of concurrence being an entanglement monotone [19].

A particular realization of these LOCC on some seriesparallel hierarchical lattices [20] has been given in Ref. [11], where the series rule is called a worst-case entanglement (WCE) strategy, since it maximizes the WCE established in a 1D chain. Here, we argue that the parallel rule is also a WCE strategy for parallel links, because it not only maximizes the average concurrence but also guarantees that the worst case is equal to the average.

On general networks, the higher-order connectivity rules produced by the SM transform may not be realizable by LOCC. They are only approximations of the true LOCC-allowing rules. Generalizing a quantum channel by including multiple entanglement links may help us understand and even determine the true rules - a difficult task to be handled by multipartite strategies [6] and QN routing [21].

Percolation thresholds.-The Bethe lattice is a typical series-parallel network where each node has the same degree $k$ [Fig. 1(a)]. $P_{\mathrm{SC}}$ and $C_{\mathrm{SC}}$ are defined as between the root and the entire boundary and can be solved exactly. Using an exact renormalization technique on the series and parallel rules (see Supplemental Material [16]), we first recover the classical threshold $p_{\text {th }}=1 /(k-1)$; whereas in ConPT we find $c_{\text {th }}=1 / \sqrt{k-1}$, and thus the ConPT threshold is always smaller, i.e., $1-\sqrt{1-c_{\text {th }}^{2}} \leq p_{\text {th }}$. Interestingly, the percolation curve of $C_{\mathrm{SC}}$ [Fig. 1(e)] exhibits not only a percolation threshold $c_{\text {th }}$ but also a saturation point $c_{\text {sat }}$ which can be solved exactly too, $c_{\text {sat }}=\sqrt{(1 / 2)^{1 / k}-(1 / 4)^{1 / k}} / \sqrt{(1 / 2)^{(k-1) / k}-(1 / 4)^{(k-1) / k}}$, an anomaly of the ConPT parallel rule (Table II) being not a smooth function. The existence of a saturation point reflects a stunning quantum advantage in Bethe lattices: with certainty one can establish a singlet that connects any node to the boundary, as long as the entanglement in each link exceeds the saturation point. This advantage cannot be revealed from any scheme based on classical percolation theory where a singlet can only be established with certainty if each link is also perfectly entangled.

If we replace $k$ by $f k+(1-f)$ in $p_{\text {th }}$ and $c_{\text {th }}$ $(0<f \leq 1)$, then $p_{\text {th }}$ and $c_{\text {th }}$ will denote the thresholds not for the original Bethe lattice but for a diluted one where $1-f$ fraction of links are randomly removed (see Supplemental Material [16]). A less-than-one $f$ can be understood as an imperfection of LOCC, and the dependence of $p_{\text {th }}$ and $c_{\text {th }}$ on $f$ thus determines the robustness of entanglement transmission under random imperfections. When $f<1 /(k-1)$, both $p_{\text {th }}$ and $c_{\text {th }}$ become unphysical because of the breakdown of the Bethe lattice structure.

Finally, Figs. 1(b)-1(d) show 2D lattices with left and right boundaries (blue dots) and possible paths connecting them (arrow lines), for which the SM transform must be used to determine the higher-order connectivity. Shown correspondingly in Figs. 1(f)-1(h) are how the spongecrossing quantities change as a function of $p$ and $c$. We find, again, that the thresholds predicted by ConPT are always smaller. Indeed, this result can be understood in an exact manner by directly comparing the series and parallel rules in Table II [22].

Critical behavior.-Percolation theory is associated with universal critical behavior near the percolation threshold. We hypothesize that ConPT as a generalization of bond percolation should also exhibit critical exponents that depend on dimensionality but not on short-range details. However, ConPT is not defined by clusters but on paths, thus lacking a suitable clusterlike definition of an order parameter. Hence, we focus solely on the thermal exponent, 


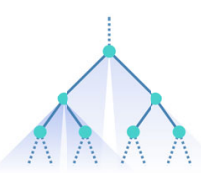

(a)

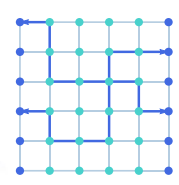

(b)

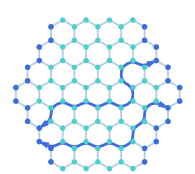

(c)

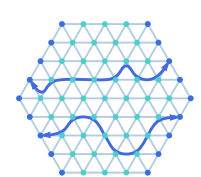

(d)
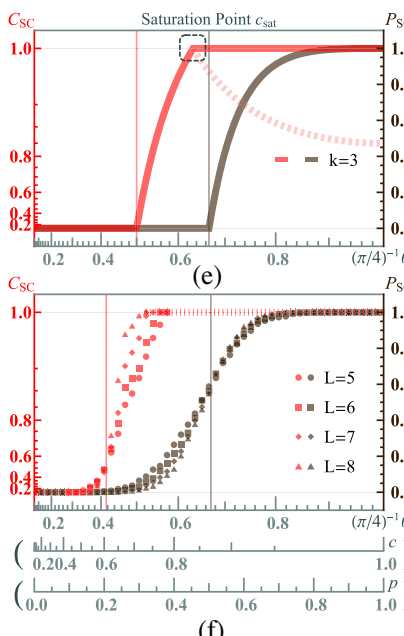

(f)
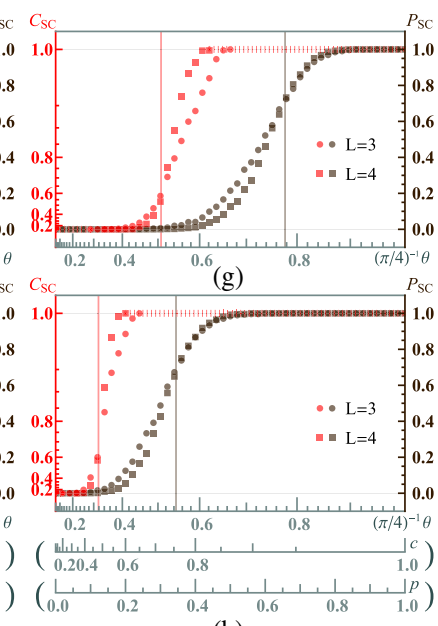

(h)

FIG. 1. Comparison between classical percolation theory and ConPT. (a) Bethe lattice (e.g., $k=3$ ). (b) Square lattice (e.g., $L=5$ ). (c) Honeycomb lattice (e.g., $L=4$ ). (d) Triangular lattice (e.g., $L=4)$. (e) For the Bethe lattice, $P_{\mathrm{SC}}=1-(1-p)^{3} / p^{3}$ yields $p_{\text {th }}=1 / 2$ for $k=3 ; C_{\mathrm{SC}}=\sin \left\{2 \cos ^{-1}\left[\left(\sqrt{1 / 4+c^{-2}}-\right.\right.\right.$ $\left.\left.1 / 2)^{3 / 2}\right]\right\}$ yields not only $c_{\text {th }}=1 / \sqrt{2}$ but also $c_{\text {sat }}$ above which the analytical solution is unphysical (red dashed), making $C_{\mathrm{SC}}=$ 1 when $c \geq c_{\text {sat }}$. (f)-(h) For the corresponding 2D lattice types (b)-(d), SM transform approximations produce $C_{\mathrm{SC}}$ (red) with respect to $c \equiv \sin 2 \theta$, compared with $P_{\mathrm{SC}}$ (brown) with respect to $p \equiv 2 \sin ^{2} \theta$ produced by standard Monte Carlo simulations. $c_{\text {th }}$ (red vertical) and $p_{\text {th }}$ (brown vertical) are determined by their finite-size crossing points.

$\nu$, which characterizes the divergence of correlation length [23]. $\nu$ is fully and universally determined by the spacial dimension but not the order parameter and therefore may be confirmed conclusively.

The most feasible way to extract $\nu$ is by finite-size analysis. Figure 2 shows how this is done for the Bethe lattice which yields mean-field exponents. Recognizing the number of layers $l$ as the shortest-path distance between the root and the boundary [23], we find $z \nu=1$ both below $c_{\text {th }}$ [Fig. 2(a)] and above $c_{\text {th }}$ [Fig. 2(b)], where $z$ is the dynamical exponent. For infinite-dimensional structures it is reasonable to expect that $l^{*} \sim \xi^{z}=\xi^{2}$ holds as a general random-walk nature [23] between the characteristic "time" $l^{*}$ and the Euclidean correlation length $\xi \sim$ $\left|c-c_{\mathrm{th}}\right|^{-\nu}$ for not only classical percolation but also ConPT. Thus $\nu=1 / 2$ is derived.

We may proceed and find other universal power laws, especially, $C_{\mathrm{SC}} \sim\left|c-c_{\mathrm{th}}\right|^{1 / 2}$ and $1-C_{\mathrm{SC}} \sim\left|c-c_{\text {sat }}\right|^{2}$ near $c_{\text {th }}$ and $c_{\text {sat }}$, respectively, independent of $k$ [Fig. 2(c)]. However, in ConPT there is no reason to fix the order parameter to be $C_{\mathrm{SC}}$ and claim that $\beta=1 / 2$. It is equally

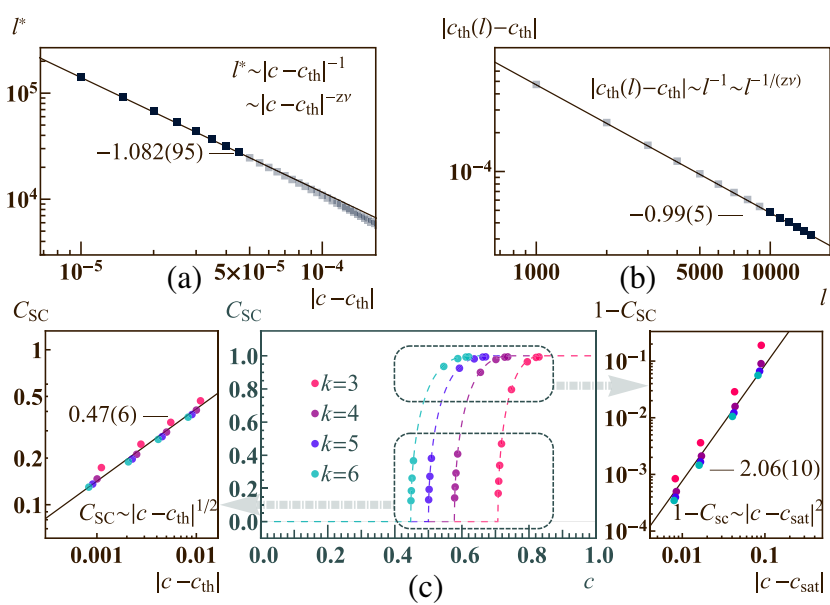

FIG. 2. Universality for the Bethe lattice. (a) Finite-size analysis of ConPT below $c_{\text {th }}=1 / \sqrt{2}(k=3)$. $C_{\text {SC }}$ follows a power law with an exponential cutoff with respect to the number of layers $l, C_{\mathrm{SC}} \sim l^{-1 / 2} \exp \left(-l / l^{*}\right)$, where $l^{*}$ diverges as a power law when approaching $c_{\text {th }}$. Numerically $z \nu=1.082(95)$ is obtained by fitting near $\left|c-c_{\text {th }}\right| \sim 10^{-5}$ (dark blue squares). (b) Finite-size analysis above $c_{\text {th }}(k=3)$. The finite-size critical threshold $c_{\mathrm{th}}(l)$ is defined as the turning point of $C_{\mathrm{SC}}$, $c_{\text {th }}(l)=\left.c\right|_{\partial^{2} C_{\mathrm{SC}} / \partial c^{2}=0}$, which deviates from $c_{\text {th }}$ as a power law with respect to $l$. Again, numerically $1 /(z \nu)=0.99(5)$ is obtained near $l \sim 10^{4}$ (dark blue squares). (c) $c_{\text {th }}$ and $c_{\text {sat }}$ for general $k$. Two universal power laws of $C_{\mathrm{SC}}$ with respect to $c$ are found by series expansions near $c_{\text {th }}$ and $c_{\text {sat }}$ and confirmed by numerical results (dots) on a finite Bethe lattice of $l=500$.

possible to let the order parameter be $C_{\mathrm{SC}}$ to some arbitrary $x$ th power near the critical threshold, but then we will have $\beta=x / 2$ unfixed.

Finite-size analysis on 2D lattices is more difficult. As shown in Figs. 1(f)-1(h), both $P_{\mathrm{SC}}$ and $C_{\mathrm{SC}}$ seem to gradually converge to a step function as the system size $L \sim \sqrt{n}$ increases, exhibiting an essential finite-size effect (despite very small $L$ because of the heavy computation needed in solving the SM transform). Here, we take advantage of a set of finite-size scaling relations first established by Kesten [24] in 2D percolation to explain the universal exponential decay of sponge-crossing probability in either the subcritical regime, given by $P_{\mathrm{SC}}\left[\mathcal{G}_{\theta}(n)\right] \sim e^{-L / \xi}, p<p_{\text {th }}$, or the supercritical regime, by $1-P_{\mathrm{SC}}\left[\mathcal{G}_{\theta}(n)\right] \sim e^{-L / \xi}, p>p_{\text {th }}$. The scaling of $Z_{\xi}[25]$ in both sub- and supercritical regimes for classical percolation is jointly plotted [Fig. 3(a)]. The thermal exponent hence obtained is close to the known exact and universal value $\nu=4 / 3$ for different 2D lattices. On the other hand, for ConPT a similar value $\nu=1.3(3)$ is obtained [Fig. 3(b)], also seemingly independent of lattice types. The 2D thermal exponents of both classical percolation and ConPT are thus not very different, hinting that the two might belong to the same universality class. This can be eventually tested if a proper definition of order parameter for ConPT is possible so that other critical exponents will be accessible. 


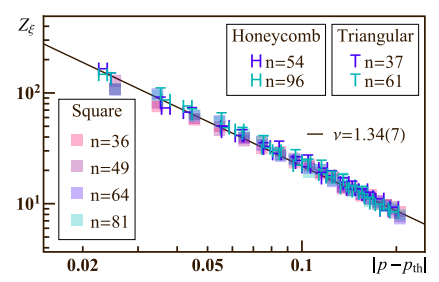

(a)

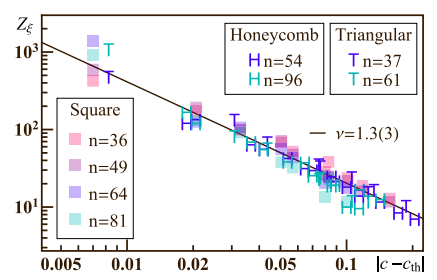

(b)
FIG. 3. Universality for 2D lattices. Finite-size analysis is performed in Figs. 1(f)-1(h). (a) For classical percolation $\nu=1.34(7)$ is known and verified. (b) For ConPT $\nu=1.3(3)$ is found. $Z_{\xi}=\left|p-p_{\text {th }}\right|^{-\nu}$ (or $\left|c-c_{\text {th }}\right|^{-\nu}$ ).

Discussion.-Our results promote the comprehension of how efficient an entanglement transmission strategy can be designed by LOCC. That being said, it is necessary to expand the theoretical framework to understand mixed states, as any realistic quantum device will unavoidably bring in thermal noise or randomly break down. Another theoretical interest here is the shift of focus of statistical theory from clusters to paths. This has been considered and explored in problems of classical directed percolation [26] and the corresponding quantum topological order models [27]. Our results suggest that path connectivity should be more general than clusterlike quantities, as the latter are always limited to probability measures yet the former is not. We hope to understand this better also for higherdimensional lattices and complex networks in the future.

We thank I. Bonamassa, R. Berkovits, H. E. Stanley, and J. Morrow for their help and useful discussions. X. M. would like to thank J. Ma, T. Yang, and Y. Xin for discussion. S. H. acknowledges the support of Israel Science Foundation, ONR, and BSF-NSF Grant No. 2019740. X. M. and S. H. are supported by DTRA Grant HDTRA-1-19-1-0016. J. G. acknowledges the support of National Science Foundation under Grant No. 2047488, and the Rensselaer-IBM AI Research Collaboration.

*jianxi.gao@gmail.com

†havlins@gmail.com

[1] A. Acín, J. I. Cirac, and M. Lewenstein, Nat. Phys. 3, 256 (2007).

[2] H. J. Kimble, Nature (London) 453, 1023 (2008); J. Biamonte, M. Faccin, and M. D. Domenico, Commun. Phys. 2, 53 (2019).

[3] R. Albert and A.-L. Barabási, Rev. Mod. Phys. 74, 47 (2002); M. Newman, A.-L. Barabási, and D. J. Watts, The Structure and Dynamics of Networks, 1st ed. (Princeton University Press, Princeton, NJ, 2006).

[4] M. A. Nielsen, Phys. Rev. Lett. 83, 436 (1999).

[5] S. Broadfoot, U. Dorner, and D. Jaksch, Europhys. Lett. 88, 50002 (2009); Phys. Rev. A 81, 042316 (2010).

[6] S. Perseguers, D. Cavalcanti, G. J. Lapeyre, M. Lewenstein, and A. Acín, Phys. Rev. A 81, 032327 (2010).
[7] M. Cuquet and J. Calsamiglia, Phys. Rev. Lett. 103, 240503 (2009).

[8] S. Perseguers, M. Lewenstein, A. Acín, and J. I. Cirac, Nat. Phys. 6, 539 (2010).

[9] M. Cuquet and J. Calsamiglia, Phys. Rev. A 83, 032319 (2011); L. Wu and S. Zhu, Phys. Rev. A 84, 052304 (2011).

[10] M. Żukowski, A. Zeilinger, M. A. Horne, and A. K. Ekert, Phys. Rev. Lett. 71, 4287 (1993); S. Bose, V. Vedral, and P. L. Knight, Phys. Rev. A 60, 194 (1999).

[11] S. Perseguers, J. I. Cirac, A. Acín, M. Lewenstein, and J. Wehr, Phys. Rev. A 77, 022308 (2008).

[12] S. Hill and W. K. Wootters, Phys. Rev. Lett. 78, 5022 (1997).

[13] H. Kesten, Commun. Math. Phys. 74, 41 (1980); J. C. Wierman, Adv. Appl. Probab. 13, 298 (1981).

[14] R. J. Duffin, J. Math. Anal. Appl. 10, 303 (1965).

[15] L. Versfeld, Electron. Lett. 6, 597 (1970).

[16] See Supplemental Material at http://link.aps.org/ supplemental/10.1103/PhysRevLett.126.170501 for a description of star-mesh transform and other results.

[17] D. J. Frank and C. J. Lobb, Phys. Rev. B 37, 302 (1988).

[18] U. Weiss, Quantum Dissipative Systems, 2nd ed., Series in Modern Condensed Matter Physics Vol. 10 (World Scientific, Singapore, 1999).

[19] G. Vidal, Phys. Rev. Lett. 83, 1046 (1999); J. Mod. Opt. 47, 355 (2000).

[20] H. D. Rozenfeld, S. Havlin, and D. ben-Avraham, New J. Phys. 9, 175 (2007); H. D. Rozenfeld and D. ben-Avraham, Phys. Rev. E 75, 061102 (2007).

[21] S. Pirandola, Commun. Phys. 2, 51 (2019); M. Pant, H. Krovi, D. Towsley, L. Tassiulas, L. Jiang, P. Basu, D. Englund, and S. Guha, npj Quantum Inf. 5, 25 (2019).

[22] Bear in mind that $1-c_{i}^{2} \equiv\left(1-p_{i}\right)^{2}$. Comparing the series rules yields $c^{2}=\prod_{i} c_{i}^{2}=\prod_{i} p_{i}\left(2-p_{i}\right) \geq$ $\left(\prod_{i} p_{i}\right)\left(2-\prod_{i} p_{i}\right)=p(2-p)$, which is proved by the subadditivity of $f(x)=\ln \left(2-e^{-x}\right)$ for $x \geq 0$. Comparing the parallel rules yields $\frac{1}{2}+\frac{1}{2} \sqrt{1-c^{2}}=$ $\prod_{i}\left(\frac{1}{2}+\frac{1}{2} \sqrt{1-c_{i}^{2}}\right)=\prod_{i}\left(1-p_{i} / 2\right) \leq \frac{1}{2}+\frac{1}{2} \prod_{i}\left(1-p_{i}\right)=$ $1-p / 2$, which is proved by the subadditivity of $f(x)=-\ln \left(1 / 2+e^{-x} / 2\right)$ for $x \geq 0$. Both inequalities further yield $1-\sqrt{1-c^{2}} \geq p$, showing the general quantum advantage independent of QN topology in both the series and parallel rules of ConPT.

[23] D. ben-Avraham and S. Havlin, Diffusion and Reactions in Fractals and Disordered Systems, 1st ed. (Cambridge University Press, Cambridge, England, 2000).

[24] H. Kesten, Commun. Math. Phys. 109, 109 (1987); C. Borgs, J. T. Chayes, H. Kesten, and J. Spencer, Random Struct. Algorithms 15, 368 (1999); P. Nolin, Ann. Probab. 36, 1748 (2008).

[25] The universal renormalization coefficient of correlation length $Z_{\xi}$ is defined by $\ln \xi=\ln Z_{\xi}+$ nonuniversal terms.

[26] H. Hinrichsen, Adv. Phys. 49, 815 (2000).

[27] M. den Nijs and K. Rommelse, Phys. Rev. B 40, 4709 (1989); H.-D. Chen and Z. Nussinov, J. Phys. A 41, 075001 (2008); G. Y. Chitov and T. Pandey, J. Stat. Mech. (2017) 043101; G. Y. Chitov, Phys. Rev. B 97, 085131 (2018); G. Y. Chitov, T. Pandey, and P. N. Timonin, Phys. Rev. B 100, 104428 (2019). 